\title{
Pulmonary arteriovenous malformations: lung transplantation as a therapeutic option
}

\author{
M. Reynaud-Gaubert**, P. Thomas*, J-Y. Gaubert***, P. Pietri*, L. Garbe**, R. Giudicelli*, \\ J. Orehek ${ }^{+}$, P. Fuentes*
}

\begin{abstract}
Pulmonary arteriovenous malformations: lung transplantation as a therapeutic option. $M$. Reynaud-Gaubert, P. Thomas, J-Y. Gaubert, P. Pietri L. Garbe, R. Giudicelli, J. Orehek, P. Fuentes. (C)ERS Journals Ltd 1999.

ABSTRACT: Multiple pulmonary arteriovenous malformations (PAVM) constitute an uncommon cause of respiratory disability. They may lead to severe hypoxaemia via right-to-left shunts and are sources of substantial mortality and morbidity. Conservative surgical resection has been proposed as the treatment of choice. More recently, percutaneous balloon or coil embolization of the feeding vessels offered an efficacious and safe alternative therapy for patients whose fistulas are too numerous to excise.

This study reports an unusual case of respiratory disability in a patient with multiple and microscopic pulmonary arteriovenous malformations who failed to respond to embolotherapy and who received a double lung transplantation with good initial outcome.

Eur Respir J 1999; 14: 1425-1428.
\end{abstract}

Direct communication between pulmonary arteries and veins via tortuous vascular spaces (pulmonary arteriovenous malformations; PAVM) is an uncommon disease. The arteriovenous malformations may be limited to the pulmonary circulation, or not as in the hereditary haemorrhagic telangiectasia, also known as Rendu-Osler-Weber syndrome occuring in $60 \%$ of cases [1]. Several mucosae and organs can be affected, even if an unequal distribution is the rule, causing occasional bleeding (epistaxis, haemoptysis, hemothorax, gastrointestinal hemorrhages) or neurological complications (embolism, brain abscess formation from septic embolism, haemorrhages). Solitary or multiple fistulae may result in significant right-to-left shunt, and lead to clinical symptoms such as dyspnoea on exertion, central cyanosis, clubbing and polycythemia. The aetiology and natural history of the disease are still poorly understood although a genetic origin (autosomal dominant pattern of transmission) has been established for the Rendu-Osler-Weber syndrome [2]. Therapy for PAVM was surgical until the advent of transcatheter embolization which now appears to be the method of choice in the centres experienced with this technique [3].

This study reports the case of a patient who presented with multiple bilateral pulmonary arteriovenous malformations leading to severe hypoxaemia, in whom multiple attempts of catheter embolization failed, who finally underwent a successful double lung transplantation.

\section{Case report}

A 27-yr-old male was referred to the authors' institution for evaluation for lung transplantation because of end-stage lung disease secondary to multiple PAVM.
*Dept of Thoracic Surgery, ${ }^{+}$Pulmonary Diseases, and **Pathology, Sainte-Marguerite Hospital; ***Dept of Radiology, La Timone Hospital, Marseille, France.

Correspondence: M.L. Reynaud-Gaubert, Service de Chirurgie Thoracique, Hôpital Sainte-Marguerite, BP 29, 13274 Marseille, Cedex 9, France, Fax: 330491744590

Keywords: Hereditary hemorrhagic telanglectasia, lung transplantation, pulmonary arteriovenous malformations

Received: March 311999

Accepted after revision June 41999

Supported by the equipment from Assistance Publique-Hôpitaux de Marseille
Diffuse PAVM had been diagnosed at the age of $25 \mathrm{yrs}$ based on clinical and radiological findings. At the time of initial presentation, the patient complained of breathlessness on exertion, palpitations, vague anterior chest pain and migraine headaches. There was neither a history of epistaxis, lower respiratory tract infection nor neurologic complications. There were no signs or symptoms to suggest extrapulmonary arteriovenous fistula. The skin and mucosa showed no telangiectasia, and a family history of telangiectasia was not present, although a 35-yr-old aunt died from cerebrovascular bleeding. Forced vital capacity (FVC) was $80 \%$ of predicted, and pulmonary diffusing capacity for carbon monoxide was reduced by $30 \%$. The baseline arterial oxygen tension $\left(\mathrm{Pa}_{\mathrm{a}} \mathrm{O}_{2}\right)$ while breathing room air was $5.4 \mathrm{kPa}$. After breathing $100 \%$ oxygen for 20 min, right-to left shunting was estimated at $>23 \%$ of cardiac output.

Opacities of uniform density, associated with linear shadows in the lower zones of both lungs were seen on postero-anterior chest radiographs (fig. 1). Contrast-enhanced spiral computed tomography (CT) confirmed the existence of multiple vascular opacities, located in both lower lobes, and showed feeding vessels of some fistulas. Brain magnetic resonance imaging examination was normal. Pulmonary angiography defined the exact feeding and draining vessels, and permitted the detection of PAVM that were judged amenable to embolization (fig. 2).

After selective catheterization of the feeding arteries, the patient underwent percutaneous coil embolization of the fistulae in seven successive sessions, one month apart. About 10 PAVM were occluded during each procedure. Mean resting $\mathrm{Pa}, \mathrm{O}_{2}$ increased to $7 \mathrm{kPa}$ after the first three sessions, but worsened again 6 months later with return to the 

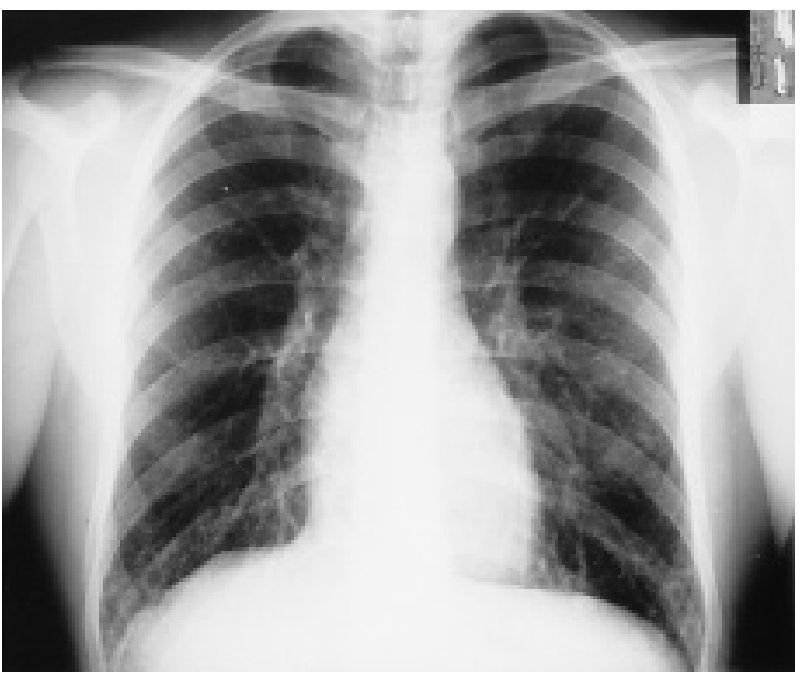

Fig. 1. - Initial plain chest radiograph showing small nodules and linear shadows in the lower lung fields.

pre-embolization values. On two occasions the patient complained of pleuretic chest pain in the $24 \mathrm{~h}$ following embolotherapy which was thought to be due to infarction of the peripheral lung to a coil, and which required high doses of analgesic drugs after the seventh procedure.

One month after the final embolization procedure, the patient was referred to the authors' institution because of increasing fatigue and shortness of breath, and two recent episodes of syncope due to shunting while walking. Physical examination at rest showed tachypnoea (28 breath$\mathrm{s} \cdot \mathrm{min}^{-1}$ ) with platypnoea, diffuse cyanosis and marked clubbing. The lungs were clear. No evidence of neurologic abnormality was detected. Blood pressure was 110/80 $\mathrm{mmHg}$ in the arms and in the legs. Pulmonary function tests were similar to the initial tests. At rest while breathing room air and lying supine, the patient had severe hypoxaemia: $P \mathrm{a}, \mathrm{O}_{2}$ was $5.7 \mathrm{kPa}$ and arterial oxygen saturation $\left(\mathrm{Sa}, \mathrm{O}_{2}\right) 76 \%$, decreasing an average of $8 \%$ when standing up; arterial carbon dioxide tension $\mathrm{Pa}_{\mathrm{a}} \mathrm{CO}_{2}$ was low (4.2 $\mathrm{kPa}$ ) and $\mathrm{pH}$ was in the normal range. $\mathrm{Sa}_{\mathrm{a}} \mathrm{O}_{2}$ decreased to

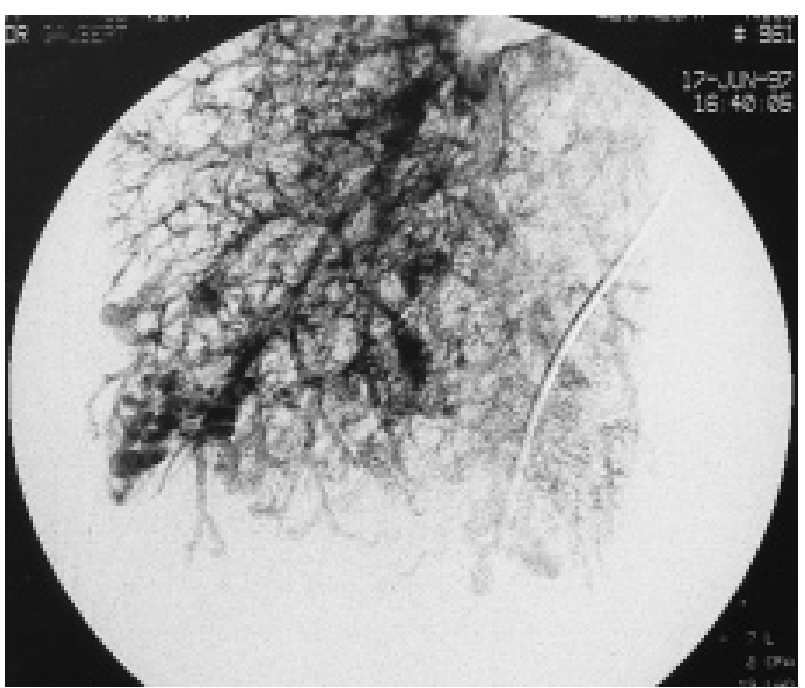

Fig. 2. - Magnified view of the right lower lung field from a pulmonary angiogram revealed numerous arteriovenous malformations.
$69 \%$ after walking $200 \mathrm{~m}$ in $4 \mathrm{~min}$. Right-to-left shunting while breathing pure oxygen was again estimated as $>23 \%$ of cardiac output. Haemoglobin concentration was 15.6 $\mathrm{g} \cdot \mathrm{L}^{-1}$ of blood and haematocrit was $52 \%$.

Chest radiography showed the presence of multiple coils in the lower lobes (fig. 3a). A follow-up CT scan of the chest showed two distal infarction areas and the disappearance of $>80 \%$ of PAVMs; the size of the remaining PAVM had markedly decreased (fig. 3b). Pulmonary haemodynamics were as follows: pulmonary artery pressure (PAP): $20 / 4 / 12 \mathrm{mmHg}$, right auricular pressure $4 \mathrm{mmHg}$, low pulmonary vascular resistance indices $(0.5 \mathrm{mmHg}$. $\mathrm{L}^{-1} \cdot \mathrm{min}^{-1} \cdot \mathrm{m}^{-2}$ ), cardiac index $7.3 \mathrm{~L} \cdot \mathrm{min}^{-1} \cdot \mathrm{m}^{-2}$. No intracardiac shunt was found, which was confirmed by contrast echocardiography.

Fibreoptic bronchoscopy was normal, while oesogastroscopy showed the presence of five angiomas without haemorrhage. Other extrathoracic vascular communications were looked for, and no lesion was detected; the patient was therefore judged to be suitable for lung transplantation.

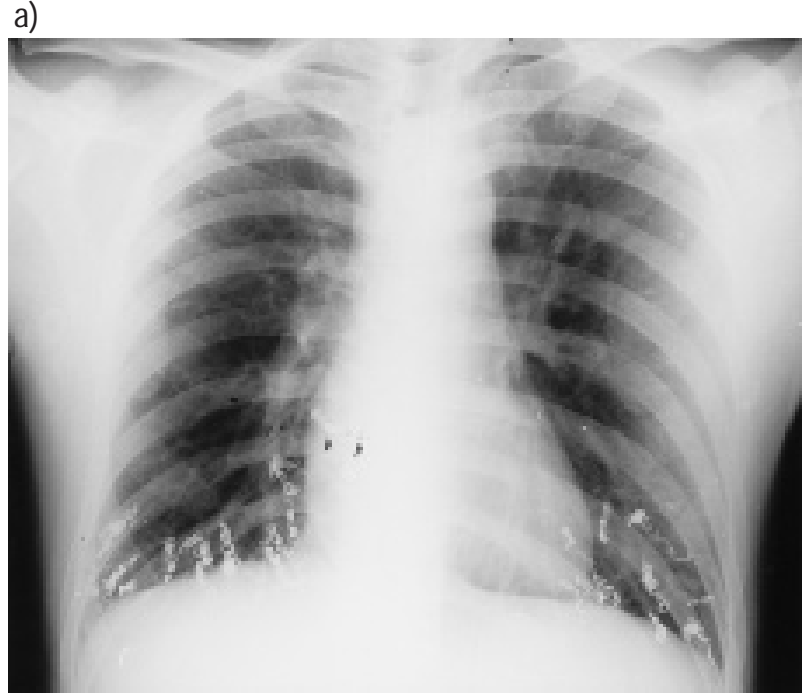

b)

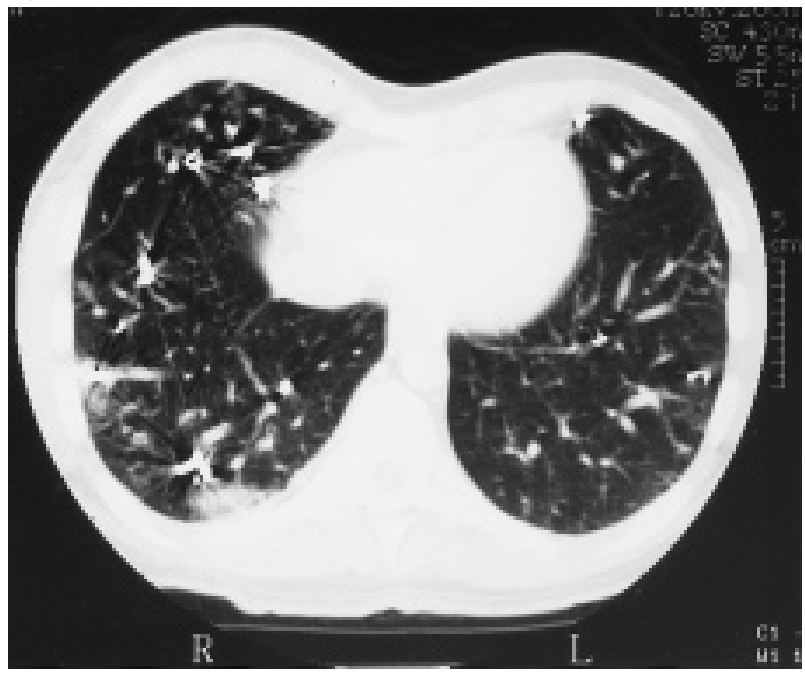

Fig. 3. - a) Postero-anterior chest radiograph, and b) computed tomography with peripheral small areas of condensation reflecting possible pulmonary infarction after coils embolization. 
Bilateral sequential lung transplantation was performed in June 1998. The cold ischemic time was $360 \mathrm{~min}$ for the right lung and $400 \mathrm{~min}$ for the left lung. At operation, careful inspection of the lung parenchyma disclosed innumerable small-sized vascular malformations puffing out the visceral pleura. Dense basal pleural effusion was also present on the right side. One of the coils used for embolizing a basal peripheral fistula penetrated the diaphragm. At the time of the basal freeing of the lung, a systemic-pulmonary vascular connection between the chest wall and lung was detected and led to a significant blood loss before it could be controlled. The immediate postoperative course was uneventful and the patient was discharged on the 35th postoperative day. Standard triple immunosuppressive therapy (cyclosporine A, azathioprine and steroid) was maintained. Eight months after transplantation, the patient was well, and respiratory status and radiological surveillance were satisfactory. Blood gas values $\left(\mathrm{Pa}, \mathrm{O}_{2}\right.$ was $11.7 \mathrm{kPa}, \mathrm{Pa}_{1} \mathrm{CO}_{2}$ was $\left.5 \mathrm{kPa}\right)$ and 6-min walk test results were normal. Specimens obtained from bronchoalveolar lavage were negative and histological sections from several transbronchial lung biopsies were representative and normal.

Pathological examination of the native lungs showed multiple and diffuse plexus of intercommunicating tortuous vessels. The venules were markedly dilated and convoluted with an irregularly thickened wall. Many fistulae abutted the visceral pleura, which became markedly thickened where it overlay the malformation (fig. 4).

\section{Discussion}

Because PAVM cause significant morbidity and mortality if lesions are untreated, radical treatment is generally mandatory as soon as PAVM is detected, even in asymptomatic patients. The aims of therapy are to reduce rightto-left shunting, and to prevent bleeding episodes and neurological events following repeated cerebral embolism. Two therapeutic options are generally considered: surgical removal or occlusion of fistulas by embolization [2].

Surgical treatment with parenchyma-sparing procedures remains the golden standard for large isolated malformations. Atypical or segmental resection is the preferred



Fig. 4. - Photomicrograph of parenchymal tissue stained by haematoxylin-eosin showing a large vein and dilated arterioles with an irregularly thickened wall. The subpleural location is characteristic. (Internal scale bar $=200 \mu \mathrm{m}$.) operative method. Surgical padding is only recommended if all contributing arteries are identified and selectively ligated. In case of central location of large fistula, lobectomy may be necessary. Video-assisted thoracic surgery has recently been advocated in order to minimize the surgical insult [4]. Finally, a surgical resection can be proposed as a complementary procedure of embolotherapy [5].

Percutaneous endovascular embolization of feeding vessels with metal coils and/or detachable balloons has recently emerged as a minimally invasive procedure and an efficient mode of treatment. Arterial occlusions have been reported in numerous series of patients, with an average success rate of $98 \%[3,6]$. A total of $>800$ PAVM occlusions performed in 252 patients was recently described in a general review [2]. Depending on patient tolerance, multiple PAVM may be embolized during a single procedure, but additional sessions may be performed if the treatment is incomplete. No periprocedural mortality has been reported to date and complications were in general self-limited [2, 3, 7]. The most common immediate complications of embolotherapy have been pleuretic chest pain, related to pulmonary infarction most likely secondary to occlusion of arteries supplying some normal lung parenchyma [7]. Air embolism occurring during the procedure has been suspected and coil migration into the systemic circulation (often due to coils being to small or too large fistula) has been rarely noted in the treatment of large fistulae. Medium term outcome frequently resulted in improvement of dyspnoea, mean shunt fraction, oxygenation and cardiac output $[8,9]$. Long-term results of this therapy have been variable in individual series. In a study based on CT scan follow-up, $96 \%$ of the embolized PAVM became either undetectable or reduced in size [10]. Some treated patients who were followed long-term rarely experienced clinical symptoms associated with recurrence of the PAVM. In this occurrence, there would be a small rate of delayed recanalization of embolized vessels and/or development of new lesions from either pulmonary artery or systemic arteries $[10,11]$.

In the current patient who suffered from multiple and bilateral PAVM, repeated staged percutaneous transcatheter embolizations seemed a logical approach. However, the number of PAVM appeared to be a limitation of the technique, as well as the nature of the disease that could lead to the recruitment of microscopic vascular malformations as soon as the macroscopic ones had been occluded.

It is likely that the clinical condition of the patient failed to respond to embolotherapy due to the presence of multiple and diffuse microfistulae which were inaccessible to catheterization. In such cases, double lung transplantation can constitute a therapeutic option. To the authors' knowledge, no case of lung transplantation in an adult patient with end-stage lung disease related to diffuse pulmonary arteriovenous malformations has been reported to date. The immediate and medium term result is satisfactory. The potential for recurrence of the disease in the donor organ is so far unknown.

\section{References}

1. Guttmacher AE, Marchuk DA, White RI. Hereditary hemorrhagic telangiectasia. N Engl J Med 1995; 333: $918-$ 924. 
2. Gossage JR, Kanj G. Pulmonary arteriovenous malformations. A state of the art review. Am J Respir Crit Care Med 1998; 158: 643-661.

3. Haitjema TJ, Overtoom ThC, Westermann CJJ, Lammers JWJ. Embolisation of pulmonary arteriovenous malformations: results and follow up in 32 patients. Thorax 1995; 50: 719-723.

4. Watanabe N, Munakata Y, Ogiwara M, Miyatake M, Nakagawa F, Hirayama J. A case of pulmonary arteriovenous malformation in a patient with brain abscess successfully treated with video-assisted thoracoscopic resection. Chest 1995; 108: 1724-1727.

5. Puskas JD, Allen MS, Moncure AC, et al. Pulmonary arteriovenous malformations: therapeutic options. Ann Thorac Surg 1993; 56: 253-258.

6. White RI, Pollak JS, Wirth JA. Pulmonary arteriovenous malformations: diagnosis and transcatheter embolotherapy. J Vasc Intervent Radiol 1996; 7: 787-804.

7. Remy-Jardin M, Wattinne L, Remy J. Transcatheter oc- clusion of pulmonary arterial circulation and collateral supply: failures, incidents and complications. Radiology 1991; 180: 699-705.

8. Chilvers ER, Whyte MKB, Jackson JE, Allison DJ, Hugues JMB. Effect of percutaneous transcatheter embolization on pulmonary function, right-to-left shunt and arterial oxygenation in patients with pulmonary arteriovenous malformations. Am Rev Respir Dis 1990; 142: 420-425.

9. Andrivet P, Lofaso F, Carette MF, Allegrini J, Adnot S. Haemodynamics and gas exchange before and after coil embolization of pulmonary arteriovenous malformations. Eur Respir J 1995; 8: 1228-1230.

10. Remy J, Remy-Jardin M, Wattinne L, Deffontaines C. Pulmonary arteriovenous malformations: Evaluation with CT of the chest before and after treatment. Radiology 1992; 182: 809-816.

11. Robertson RJH, Robertson IR. Pulmonary arteriovenous malformations. Thorax 1995; 50: 707-708. 